\title{
Haralick texture and invariant moments features for breast cancer classification
}

\begin{abstract}
Classification of breast cancer is essential in determining the type of treatment that should be applied. Thus, a Computer Aided Diagnosis (CADx) may assist radiologists in making appropriate decision based on the classification results. In this paper, the classification is divided into two categories; to classify the cancer into benign and malignant (two classes) and to classify the character of the background tissue either fatty, glandular or dense (multi class). The Haralick texture features and $\mathrm{Hu}$ Invariants moments were proposed as the features extraction. There are three phases conducted in this study. The first phase is the preprocessing phase. This is followed by the features extraction phase where combination of moment based features with addition of four features was proposed. The final phase is the classification phase by using SVM classifiers. Results obtained shows that the accuracy of the proposed features are $90.5 \%$ and $77.5 \%$ for two classes and multi class respectively.
\end{abstract}

Keyword: Breast cancer; Classification; Haralick texture; Invariants moments 